\title{
Animal Virus
}

National Cancer Institute

\section{Source}

National Cancer Institute. Animal Virus. NCI Thesaurus. Code C14181.

An animal pathogen that is a virus. 